\title{
ANÁLISE DO PERFIL DA SAÚDE CARDIOVASCULAR DE FREQÜENTADORES DO PARQUE DO POVO DE PRESIDENTE PRUDENTE - SP
}

Maria Beatriz Rigo Bonilha, Felipe Ribeiro, Mariana Graça Gutierrez, Gezilaine Rodrigues Torres, Daniela Alari Chedid, Bruno Massayuki Makimoto Monteiro, Aline Ferreira Lima Gonçalves, Ana Alice Soares dos Santos, Thais Roque Giacon, Natália Turri da Silva, Aline Fernanda Barbosa Bernardo, Luiz Carlos Marques Vanderlei

Universidade Estadual Paulista - UNESP, Departamento de Fisioterapia, Pró-Reitoria de Extensão Universitária PROEX, Presidente Prudente, SP. e-mail: bibi_bonilha07@hotmail.com

\section{RESUMO}

Os hábitos de vida são responsáveis pelo aumento de indivíduos com hipertensão arterial (HA) e com doenças cardiovasculares (DCV), e a atividade física (AF) é um meio de controle e prevenção de DVC e HA. Este estudo teve como objetivo avaliar o perfil cardiovascular dos frequentadores do Parque do Povo de Presidente Prudente. 87 indivíduos $(56,31 \pm 12,83$ anos) responderam a questionários sobre a presença de DCV, HA e recomendação médica para AF e tiveram, em seguida, sua pressão arterial mensurada. Dos 87 indivíduos abordados, 17,24\% são cardiopatas e apenas $20 \%$ deles realizam exercícios por recomendação médica, 40,23\% são hipertensos e desses, 48,57\% possuem recomendação para as atividades. Tais resultados mostram uma alta taxa de hipertensos seguida por uma menor de cardiopatas, porém uma taxa muito baixa de recomendação médica para AF, o que alerta que a não supervisão pode ser perigosa por se tratar de uma população de risco.

Palavras-chave: Doenças Cardiovasculares, Hipertensão, Atividade Física, Saúde Pública, Local Público.

\section{PROFILE ANALYSIS OF THE CARDIOVASCULAR HEALTH OF PARQUE DO POVO USERS OF PRESIDENTE PRUDENTE-SP}

\begin{abstract}
Life habits are responsible for the increase in individuals with arterial hypertension (AH) and cardiovascular disease (CVD), and physical activity (PA) is a means of control and prevention of CVD and $\mathrm{AH}$. This study aimed to evaluate the cardiovascular profile of the users of the Parque do Povo of PresidentePrudente. 87 subjects (56.31 \pm 12.83 years) completed questionnaires about the presence of CVD, AH and medical recommendation for PA and then had their blood pressure measured. Of the 87 individuals approached, $17.24 \%$ are heart disease and only $20 \%$ of them perform exercises on a medical recommendation, $40.23 \%$ are hypertensive and of these, $48.57 \%$ have a recommendation for the activities. These results show a high rate of hypertension followed by a minor heart disease, but a very low rate of medical recommendation for PA, which warns that no supervision can be dangerous because it is a population at risk.
\end{abstract}

Keywords: Cardiovascular Deseases, Hypertension, Physical Activity, Public Health, Public Place. 


\section{INTRODUÇÃO}

As mudanças no perfil demográfico e epidemiológico das populações tiveram como consequência maior exposição dos indivíduos aos fatores de risco (FR) relacionados às doenças cardiovasculares (DCV), tornando-as primeira causa de morte no mundo $^{1}$, e entre esses FR desenvolvidospelos hábitos de vida modernos está a hipertensão arterial sistêmica (HAS).

A HAS é uma condição clínica multifatorial $^{2}$ caracterizada pela presença de valores de pressão arterial sistólica (PAS) igual ou superior a $140 \mathrm{mmhg}$ e pressão arterial diastólica (PAD) igual ou superior a 90 $\mathrm{mmHg}^{3}$, e entre os FR para DCV é o mais prevalente no Brasil ${ }^{4}$, podendo resultar no desenvolvimento de aterosclerose, ataques cardíacos, insuficiência cardíaca, derrame cerebral e insuficiência renal ${ }^{5}$.

O tratamento para controle da HAS inclui, além da utilização de medicamentos, a modificação de hábitos de vida ${ }^{6}$, entre elas a prática regular de atividade física (AF).

Estudos demonstram reduções significativas da PAS e PAD com a prática de exercícios aeróbicos, sendo esta redução mais expressiva em hipertensos ${ }^{7}$. A diminuição da PA com a realização de $\mathrm{AF}$ tem sido evidenciada nos dois sexos, parecendo não depender de outros fatores, como perda de peso, e tem magnitude semelhante à observada com 0 tratamento medicamentoso ${ }^{8}$.

A prática de AF não supervisionada, a qual é realizada por uma grande quantidade de pessoas principalmente em áreas abertas e no tempo livre, também exerce influências positivas para hipertensos, com reduções significativas da PA, além da melhora da aptidão física ${ }^{9}$. Dentre as $A F$ realizadas no tempo livre, andar aparece como a mais comum, sendo realizada por metade das pessoas que se exercitam ${ }^{10}$, principalmente em praças e parques públicos ${ }^{11}$.

Pela escassez na literatura de trabalhos que abordem indivíduos que realizam AF não supervisionada em seu tempo livre, principalmente no âmbito cardiovascular, o objetivo desse estudo foi avaliar o perfil dos freqüentadores do Parque do Povo de Presidente Prudente em relação à presença de DCV e HA.

\section{METODOLOGIA}

Trata-se de um estudo descritivo transversal realizado no Parque do Povo de Presidente Prudente, uma área verde extensa para o lazer, onde durante a semana é frequentada por um grande número de indivíduos como "academia a céu aberto", praticando exercícios físicos como corrida, caminhada, entre outros.

A amostra foi constituída pela população frequentadora deste local, com as 
coletas realizadas quinzenalmente nos meses de abril e maio de 2014, nos horários de maior movimentação (às $7 \mathrm{~h}$ e às $18 \mathrm{~h}$ ) por alunos do curso de fisioterapia participantes do "Projeto Hipertensão do Setor de Reabilitação Cardíaca da FCT/UNESP Campus de Presidente Prudente". Todos os procedimentos deste estudo foram aprovados pelo Comitê de Ética em Pesquisa da Instituição(2013/331954).

Foi montado um posto de coletas, no centro do parque, para que os frequentadores fossem abordados durante a prática de seus exercícios, com a indagação sobre o interesse em realizar a mensuração da pressão arterial. Em resposta afirmativa, primeiramente os voluntários respondiam a um questionário, abrangendo além de dados para caracterização da amostra (como sexo e idade), a presença de doença cardiovascular, hipertensão arterial e recomendação médica para a realização da atividade física. Para os pacientes hipertensos, ainda foi questionado o tempo do diagnóstico clínico e se era feito o uso de algum medicamento para controle da pressão arterial, caso fizesse uso quais os medicamentos utilizados.

A segunda parte da coleta constituiuse pela mensuração da pressão arterial pelo método indireto ${ }^{12}$, com o uso de um estetoscópio e um esfigmomanômetro aneróide no membro superior esquerdo, após os indivíduos permanecerem cinco minutos sentados em repouso, sendo realizado no membro contralateral apenas em casos que o lado esquerdo fosse contraindicado. Ao final da coleta, todos os voluntários receberam um folder contendo informações sobre a hipertensão arterial e seus FR.

Para análise dos dados, foi utilizada estatística descritiva para caracterização da amostra e os dados serão apresentados com os valores expressos em média, desviopadrão e porcentagem.

\section{RESULTADOS}

A amostra estudada foi composta por 87 indivíduos freqüentadores do Parque do Povo de Presidente Prudente, sendo 47 homens e 40 mulheres, com a faixa etária média de $56,31 \pm 12,83$ anos e com PAS média de $132,98 \pm 18,81 \mathrm{mmHg}$ e PAD de $82,53 \pm 11,02 \mathrm{mmHg}$.

Dentre eles, $17,24 \%(n=15)$ possuem diagnóstico clínico de doença cardiovascular, descritas na tabela 1 , sendo a maior incidência para insuficiência coronariana e a presença de arritmias de base. 
Tabela 1. Doenças cardiovasculares apresentadas pela população estudada.

\begin{tabular}{ccc}
\hline \hline Doença Cardiovascular & N & \% \\
\hline \hline Insuficiência Coronariana & 3 & $20 \%$ \\
Arritmia de Base & 3 & $20 \%$ \\
Valvulopatia & 2 & $13,33 \%$ \\
Insuficiência Cardíaca & 1 & $6,67 \%$ \\
Ateroma & 1 & $6,67 \%$ \\
AVE & 1 & $6,67 \%$ \\
Não responderam & 4 & $26,66 \%$ \\
\hline \hline
\end{tabular}

Legenda: $\mathrm{AVE}=$ Acidente vascular encefálico.

Do total, $40,23 \% \quad(n=35)$ são hipertensos, e entre os cardiopatas este número é de $53,33 \% \quad(n=8)$. Todos os hipertensos fazem uso de medicação para controle da PA, sendo os mais utilizados beta-bloqueador seletivo, antagonista do receptor (tipo AT1) da angiotensina II e diurético.

Tabela 2. Tempo de diagnóstico da hipertensão arterial

\begin{tabular}{ccc}
\hline \hline Tempo de Diagnóstico & $\mathrm{n}(\mathbf{3 5})$ & $\%$ \\
\hline \hline Menos de um ano & 3 & $8,57 \%$ \\
De um a cinco anos & 6 & $17,14 \%$ \\
Mais de cinco anos & 26 & $74,29 \%$ \\
\hline \hline
\end{tabular}

Quanto à realização de $\mathrm{AF}$ por médica e entre os hipertensos, $48,57 \%$ recomendação médica, 27 (31,03\%) do total, $(n=17)$.

entre os cardiopatas apenas $20 \% \quad(n=3)$ realizam exercícios físicos por recomendação 


\section{DISCUSSÃO}

Os resultados apontam que mais de $40 \%$ dos frequentadores do Parque do Povo são hipertensos e ainda que $17,24 \%$ possuem algum problema cardíaco, sendo que, entre os cardiopatas, $53,33 \%$ também possuem diagnóstico clínico de HAS. Em relação ao tempo de diagnóstico clínico da HAS, quase dois terços da população entrevistada o obtiveram a mais de cinco anos. Quanto à orientação para prática da $\mathrm{AF}, 80 \%$ dos cardiopatas e um pouco mais da metade dos hipertensos a realizam sem recomendação médica e/ou orientação.

Esses dados sugerem que é alta a incidência de HAS nessa população, assim como o número de indivíduos que realizam AF sem recomendação, inclusive entre os cardiopatas.

Dessa amostra que frequenta $o$ Parque do Povo para a prática de AF 40,23\% são hipertensos. Inquéritos de base populacional realizados em algumas cidades brasileiras mostram uma prevalência da HAS em adultos de $22,3 \%$ a $43,9 \%{ }^{13}$, assim a porcentagem de hipertensos encontrada em nosso estudo está dentro dos valores encontrados em outras localidades.

Forjaz et al. verificou que $12 \%$ da população frequentadora de um parque na cidade de São Paulo era hipertensa ${ }^{14}$, um número muito menor do que o encontrado no nosso estudo. De acordo com o Ministério da Saúde ${ }^{15}$, de 2006 a 2009, a porcentagem de brasileiros hipertensos saltou de 21,6\% para $24,4 \%$, podendo explicar a maior prevalência desse estudo e ainda, há uma baixa adesão de $\mathrm{AF}$ apenas para prevenção de DCV. Por essa razão, os indivíduos que realizam a sua prática estão acometidas por algumas patologias, principalmente cardiovasculares.

Abordando outros FR para DCV, e também para a HA em frequentadores de um parque público da cidade de São Paulo, Koikeet $\mathrm{al}^{11}$. constataram que os fatores mais prevalentes foram o tabagismo e histórico familiar de DCV, além da relação cinturaquadril alterada. Não foi questionado sobre a presença de HAS neste estudo, porém mais de $5 \%$ da população entrevistada possuía PA alterada.

A grande maioria apresentou o diagnóstico clínico de HAS a mais de cinco anos, essa aspecto é um diferencial do nosso estudo em relação aos demais que abordaram este tema ${ }^{14}$.Contrário aos achados, Forjaz etal. encontraram apenas 9\% de indivíduos com diagnóstico clínico de $\mathrm{DCV}^{13}$. Tal percentual pode ser sido menor, visto que os voluntários não conheciam sua real condição de saúde, $7 \%$ dos participantes que disseram não ter problemas de saúde tomavam medicamentos relacionados ao coração, e além disso, $16 \%$ dos indivíduos que se consideravam saudáveis e não tomava 
medicamentos apresentavam sintomas freqüentes de DCV, como tontura, dor no peito e fadiga ${ }^{14}$, que podem indicar a presença de problemas cardíacos ${ }^{16,17}$.

Sobre a recomendação médica para a prática de $\mathrm{AF}$, observamos que apenas $20 \%$ dos cardiopatas foram recomendados a realizar esta atividade, com um percentual um pouco maior para os hipertensos (48,57\%). Estes baixos índices de recomendação também foram encontrados no estudo de Koike etal., no qual apenas $17 \%$ dos entrevistados realizavam AF conforme recomendações recebidas. A prática de AF não supervisionada e não orientada pode não trazer benefícios no controle dos FR para a doença cardiovascular. Ou pior, pode expor o praticante a um evento súbito durante a realização do exercício físico.

Em relação às limitações do estudo podemos referir o tamanho da amostra que se encontra reduzido ao comparar-se com outros estudos de mesma natureza ${ }^{10,14}$, além disso, não foram questionados FR relacionados a HAS, como o tabagismo, consumo diário de sal e obesidade, os quais permitiriam uma melhor conscientização da população.

Este estudo possui implicações clínicas para profissionais que trabalham com saúde pública na cidade, pois os resultados mostram que o índice de hipertensos que frequentam o Parque do Povo de Presidente
Prudente é alto, assim como o de cardiopatas ao comparar-se com outros estudos ${ }^{11,14}$, e um número extremamente alto destes indivíduos realizam AF sem recomendação médica, sendo de fundamental importância a implementação de um profissional da área da saúde neste local para aconselhamento da população.

A partir dos achados, conclui-se que grande parte da população que realiza atividades físicas não supervisionadas no Parque do Povo de Presidente Prudente possui diagnóstico de HAS, e uma menor parte de DCV, e apenas $31,03 \%$ possuem recomendação médica para a atividade praticada, dados que alertam o alto risco de ocorrência de eventos súbitos, considerando que essa população já é de alto risco devido ao diagnóstico clínico que possuem.

\section{REFERÊNCIAS}

1. Lessa I. Doenças crônicas nãotransmissíveis no Brasil: um desafio para complexa tarefa da vigilância. CienSaudeColet 2006; 9(4):931-46. DOI http://dx.doi.org/10.1590/S141381232004000400014.

2. Sociedade Brasileira de Cardiologia. V Diretrizes Brasileiras de Hipertensão. ArqBrasCardiol. 2006:1-48. DOI http://dx.doi.org/10.1590/S0066782X2007001500012.

3. Neder MM, Borges AAN. Systemic hypertension in Brazil: how much have we improved our knowledge about its epidemiology? RevBrasHipertens 2006; 13(2): 126-33. 
4. Miranda RD, Perrotti TC, Bellinazzi VR, Nóbrega TM, Cendoroglo MS, Toniolo Neto J. Hipertensão arterial no idoso:peculiaridades na fisiopatologia, no diagnóstico e no tratamento. RevBrasHipertens2002; 9(3):293-300.

5. Filho JGB, Bagnara IC. Doenças cardiovasculares e atividade física. EFDeportes.com, Revista Digital2011; 15(154).

6. PéresDS, MagnaJM, VianaLA. Portador de hipertensão arterial: atitudes,crenças, percepções, pensamentos e práticas. Rev SaúdePública 2003; 37(5):635-42. DOI http://dx.doi.org/10.1590/S003489102003000500014.

7. Cornelissen VA, Fagard RH. Effects of endurance training on blood pressure, blood pressure-regulating mechanisms, and cardiovascular risk factors. Hypertension 2005; 46:667-75. DOI http://dx.doi.org/10.1161/01.HYP.00001842 25.05629 .51 .

8. Cleroux J, Feldman RD, Petrella RJ. Lifestyle modifications to prevent and control hypertension. 4. Recommendations on physical exercise training. Canadian Hypertension Society, Canadian Coalition for High Blood Pressure Prevention and Control, Laboratory Centre for Disease Control at Health Canada, Heart and Stroke Foundation of Canada. CMAJ. 1999; 160: S21-8.

9. Farinatti PTV, Oliveira RB, Pinto VLM, Monteiro WD, Francischetti E. Programa domiciliar de exercícios: efeitos de curto prazo sobre a aptidão física e pressão arterial de indivíduos hipertensos. Arq. Bras. Cardiol. 2005;

84(6):473-9.

DOI http://dx.doi.org/10.1590/S0066782X2005000600008.

10. Mello MT, Fernandez AC, Tufik S. Levantamento epidemiológico da prática de atividade física na cidade de São Paulo.
RevBrasMed Esporte 2000; 6(4):119-24. DOI http://dx.doi.org/10.1590/S151786922000000400003.

11. Koike DC, Nascimento VC, Zucco RC, Galimbertti TM, Marques TM, Wichi RB. Avaliação de fatores de risco cardiovascular em praticantes de atividade física não orientada. Revista Mackenzie de Educação Física e Esporte 2008; 7(3): 189-94.

12. Pierin AMG, Alavarce DC, Lima JC, Mion Jr. D. A medida indireta da pressão arterial: como evitar erros. Rev Bras Hipertens 2000; 7(1):31-8.

13. Sociedade Brasileira de Hipertensão, Sociedade Brasileira de Cardiologia, Sociedade Brasileira de Nefrologia. V Diretrizes Brasileiras de Hipertensão Arterial. Sociedade Brasileira de Hipertensão, Sociedade Brasileira de Cardiologia, Sociedade Brasileira de Nefrologia. ArqBrasCardiol 2007; 89: e24-e79.

14. Forjaz CLM, Tinucci T, Bartholomeu T, Fernandes TEM, CasagrandeV,Massucato JG. Avaliação do Risco Cardiovascular e da Atividade Física dos Freqüentadores de um Parque da Cidade de São Paulo. ArqBrasCardiol. 2002; 79 (1): 35-42.

15. Ministério da Saúde (BR), Secretaria de Atenção à Saúde, Política Nacional de Humanização da Atenção e Gestão do SUS. Acolhimento e classificação de risco nos serviços de urgência. Brasília: Ministério da Saúde, 2009.

16. American College Sports Medicine. ACSM's guidelines for exercise testing and prescription.Baltimore: Williams \& Wilkins, 2000.

17. American College of Sports Medicine, American Heart Association. Recommendations for cardiovascular screening, staffing, and emergency policies at health/fitness facilities. Med Sci Sports Exerc 
1998;

1009-18.

DOI http://dx.doi.org/10.1161/

01.CIR.97.22.2283.

Recebido para publicação em 12/08/2014

Revisado em 10/09/2014

Aceito em 12/09/2014 